\title{
EN MEMORIA DEL DR. BARRY R. SINERVO "DR. LIZARDO" (1960-2021) IN MEMORY OF DR. BARRY R. SINERVO "DR. LIZARDO" (1960-2021)
}

\author{
Fausto R. Méndez de la Cruz ${ }^{1 *}$, Natalia Fierro Estrada² ${ }^{2}$ Norberto Martínez Méndez ${ }^{3} \&$ Rafael Alejandro \\ Lara-Resendiz ${ }^{4}$ \\ ${ }^{1}$ Instituto de Biología, UNAM. A.P. 70-153. C.P. 04510, Ciudad de México, México. \\ ${ }^{2}$ Laboratorio de Recursos Naturales, Unidad de Biotecnología y Prototipos, Facultad de Estudios Superiores Iztacala, Universidad Nacional Autónoma \\ de México, Estado de México, México. Facultad de Estudios Superiores, Iztacala, Av de los Barrios S/N. Iztacala Estado de México. \\ ${ }^{3}$ Laboratorio de Bioconservación y Manejo, Departamento de Zoología. Escuela Nacional de Ciencias Biológicas, IPN, CdMx, México. \\ ${ }^{4}$ Consejo Nacional de Investigaciones Científicas y Técnicas (CONICET), Instituto de Diversidad y Ecología Animal (IDEA) y Universidad Nacional \\ de Córdoba, Facultad de Ciencias Exactas, Físicas y Naturales, Centro de Zoología Aplicada, Córdoba, Argentina \\ "correspondence:faustomendez6@gmail.com \\ Received: 2021-04-19. Accepted: 2021-04-28.
}

Barry Sinervo fue un investigador que impactó al mundo de la biología por su capacidad de entender los problemas de manera integral y por su elocuencia en las conferencias y cursos. Era capaz de integrar la información generada por sus investigaciones con la teoría de diversas disciplinas, impactando y sembrando una semilla de conocimiento en cada uno de los que tuvimos la oportunidad de conocerlo.

Barry nació en Port Arthur, Ontario, Canadá. Se educó en la Universidad de Dalhousie, Nueva Escocia, en donde obtuvo la licenciatura en biología/matemáticas en 1982. Posteriormente obtuvo el doctorado en la Universidad de Washington en Seattle en 1988. Comenzó a trabajar en la Universidad de California, Santa Cruz en 1997 y fue reconocido con el nombramiento de Profesor distinguido de Ecología y Biología Evolutiva en 2010.

Desde su niñez, Barry mostró un gran interés por los reptiles, principalmente las lagartijas, a pesar de ser muy escasas en su tierra natal. Su afición se convirtió en una brillante profesión y le llevó a tener grandes aportes científicos durante su carrera, teniendo como modelo principal las lagartijas, por eso se hizo llamar Dr. Lizardo (a simple country lizard doctor, según sus propias palabras). Sin embargo, durante su vida profesional realizó importantes contribuciones sobre plantas, peces, mamíferos, anfibios y reptiles en diversas partes del mundo. En el campo siempre se proponía superar sus propios registros, que hacían su ambiente competitivo y agradable; número de lagartijas capturadas por día, número de especies observadas en una expedición, etc. eran parte de los retos cotidianos. Además, musicalizaba el trabajo en campo con canciones, adecuando las

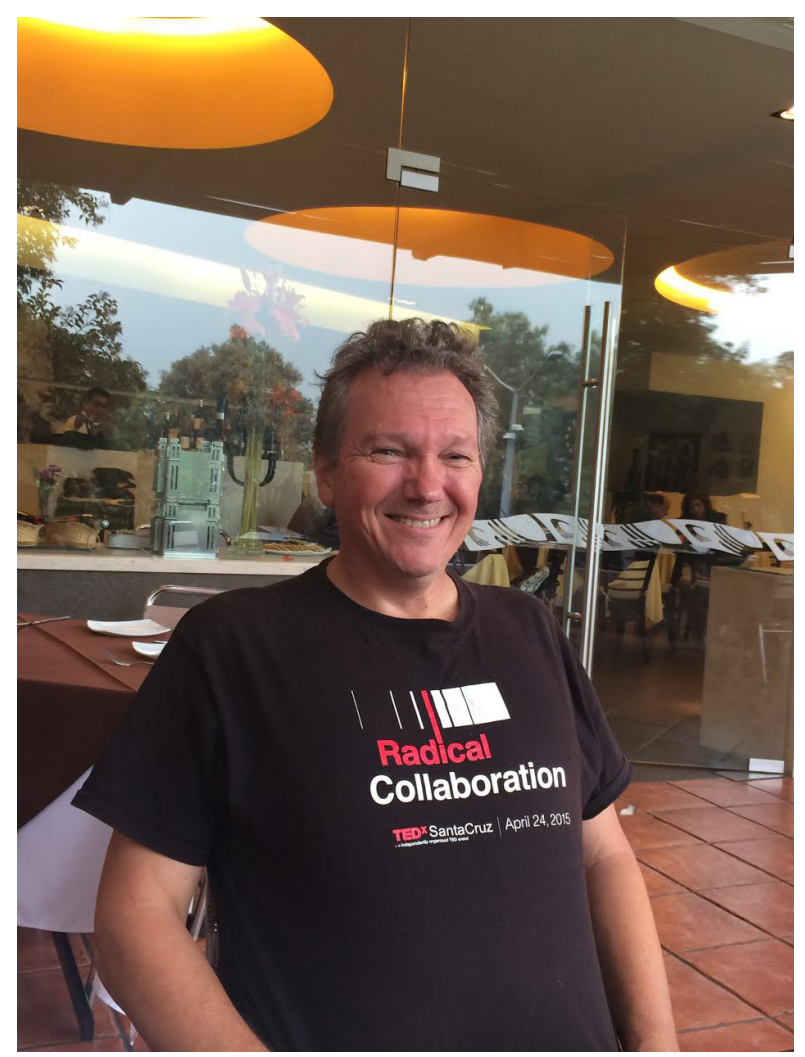

Figure 1. Dr. Barry Sinervo at Centro Cultural Universitario, UNAM. Photo: Fasuto A. Mendez.

Figura 1. Dr. Barry Sinervo en el Centro Cultural Universitario, UNAM. Foto: Fasuto A. Mendez. 


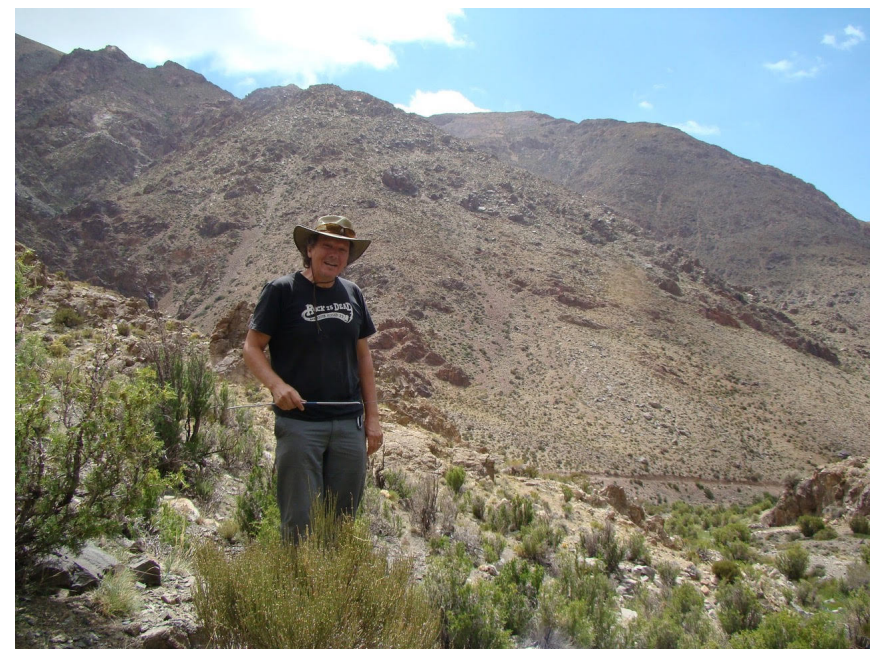

Figure 2. Barry Sinervo doing fieldwork at San Juan, Argentina (December 2011). Photo: Rafael Lara.

Figura 2. Barry Sinervo haciendo trabajo de campo en San Juan, Argentina (Diciembre 2011). Foto: Rafael Lara.

letras a las vivencias diarias. Siempre mantenía un gran sentido del humor y parte de las actividades principales durante los días en campo era contemplar los espectaculares amaneceres o atardeceres. Combinar las actividades de campo con la diversión era la dinámica garantizada al trabajar con Barry, además de disfrutar por las noches de buenas cepas de Pinot Noir o Malbec.

Barry fue un ecólogo evolutivo y del comportamiento prominente que publicó artículos que impactaron al mundo, ya que su visión de la investigación no tenía límites y percibía inmediatamente un mapa conceptual de una manera global y multidisciplinaria. Una de las investigaciones que revolucionó la ciencia fue directamente derivada de un proyecto desarrollado en "Lizard Land", una región nombrada así por él, ubicada a $130 \mathrm{~km}$ al este de Santa Cruz, en donde observó que existía un comportamiento particular de los ejemplares de la lagartija de costado manchado, Uta stansburiana, dependiendo del color que mostraban en la garganta. Este descubrimiento de Sinervo y Lively intitulado "The rock-paper-scissors game and the evolution of alternative male strategies" fue publicado en 1996 en Nature, el cual impactó al mundo de la biología por dar una perspectiva diferente de la forma en que ocurre la interacción de individuos de una misma población en función de los diferentes colores.

Durante 30 años consecutivos, Dr. Lizardo estudió los diferentes morfos cromáticos que permitieron entender una dinámica diferente en la especiación, las diferentes estrategias en la historia de vida dentro de una misma población y, de manera sobresaliente, la dinámica en la teoría de juegos, desde la perspectiva de la biología.

En México nació otro de sus más grandes aportes a la ciencia durante 2008 y 2009 cuando realizaba trabajo de campo con colaboradores mexicanos, pudo encontrar un mecanismo que explicaba cómo las poblaciones de reptiles, principalmente de lagartijas, se ven afectadas por los efectos del cambio climático. El estudio de este mecanismo le permitió crear una base conceptual y teórica para medir y estimar las extinciones en reptiles. Esto lo llevó a consolidar un equipo de colaboradores en los cinco continentes para poner a prueba sus nuevas teorías y estimar el riesgo de extinción de los reptiles alrededor del mundo. Este primer esfuerzo se vio reflejado en una publicación en 2010 en la revista Science titulada "Erosion of lizard diversity by climate change and altered thermal niches". Este primer acercamiento fue enriqueciéndose con el paso de los años con diversos aportes teóricos y técnicos que han logrado integrar otros aspectos de la fisiología, desempeño y demografía de los reptiles. Sin embargo, estas metodologías tienen el potencial de poder adaptarse a otros organismos ectotérmicos, como los anfibios, como lo demostró en publicaciones y proyectos recientes con salamandras, y particularmente, con el ajolote mexicano Ambystoma mexicanum.

Derivado de estos estudios en Barry surgió la imperiosa necesidad de concientizar a la humanidad sobre los efectos del cambio climático acelerado sobre la diversidad biológica. En este aspecto él se convirtió en un difusor incansable de la protección del medio ambiente y de la necesidad que tiene la humanidad para controlar las emisiones de gases de efecto invernadero. En 2014, Sinervo ayudó a establecer el Instituto para el Estudio de Impactos Climáticos Ecológicos y Evolutivos (ISEECI por sus siglas en inglés) aprovechando el Sistema de Reservas Naturales de la Universidad de California para estudiar cómo el cambio climático afectará los ecosistemas de California y estados limítrofes.

Barry era un excelente orador y sus conferencias y pláticas siempre lograban contagiar, a todos los que lo escuchaban, de su amor a la naturaleza y del entusiasmo que debemos poner para lograr un mundo mejor. Tuvo un gran impacto científico no solo con sus publicaciones, sino también a través de sus clases y los talleres que organizó en países de todo el mundo. En sus clases rara vez usaba notas y su presentación parecía espontánea. Un día de enseñanza de Sinervo podría valer un semestre de otros profesores. Era el tipo de profesor cuya creatividad extravagante rara vez se ve en un entorno académico. Entre 1993 y 2021 fue profesor de ecología conductual y evolutiva, teoría de juegos, 
genética de poblaciones, ecología fisiológica y herpetología. En esta última, el trabajo de campo se realizaba en salidas épicas a las cercanías de Santa Cruz, a Lizard Land o al Desierto de Mojave, donde de manera apasionada y motivacional daba cátedra de biología. Debido a su gran entusiasmo por la ciencia, historia natural, evolución y herpetología, fue un profesor sumamente carismático y querido en la universidad, que motivó y cautivó a 31 generaciones de biólogos.

Barry fue un excelente científico, siempre atento a colaborar $\mathrm{y}$ a recibir preguntas o peticiones de trabajo que solía responder las peticiones casi inmediatamente, inmediatamente con una idea propositiva. Siempre iba más allá de lo que se le planteaba, sus respuestas eran del tipo: "también podemos hacer tal cosa", "podemos estudiar tal lagartija", "con gusto doy tal charla" y "¿En qué puedo ayudar?". Barry no sólo nos dejó grandes aportes en el estudio de las lagartijas, también nos dejó grandes enseñanzas sobre la colaboración y el trabajo en equipo aún a la distancia y sobre todo la importancia de compartir el conocimiento con todo aquel interesado, lo que le llevó a tener múltiples colaboraciones.

El laboratorio de Sinervo atrajo a una incalculable cantidad de estudiantes y colaboradores provenientes de todo el mundo, con quienes realizó investigaciones multinacionales. Particularmente, asesoró a 15 estudiantes de doctorado y por su laboratorio transitaron 17 posdoctorantes. Su bibliografía contiene evidencia de su éxito como científico y colaborador en múltiples campos de investigación y diversos sistemas biológicos. Tiene alrededor de 150 publicaciones (130 artículos en revistas y 16 capítulos de libros), las cuales, de acuerdo con google académico, han sido citadas $\sim 16,200$ veces (abril/2021). Dentro de su producción destacan siete artículos en Science, dos en Nature, cinco en PNAS, 14 en Evolution y ocho en The American Naturalist. Esta última en 2004 premió como el mejor artículo a "Behavioral drive versus behavioral inertia in evolution: a null model approach" en colaboración con R. B. Huey y P. E. Hertz; también Copeia en 2012 premió al mejor artículo "Rapid formation of reproductive isolation between two populations of side-blotched lizards, Uta stansburiana" con A. Corl y L. Lancaster. Uno de sus libros más citados es "Evolutionary games in natural, social and virtual worlds" publicado con D. Friedman (2016). Los principales coautores en sus contribuciones son Donald Miles, Raymond Huey, Jonathan Losos, Jean Clobert, Erik Svensson y Lesley Lancaster, aunque tuvo colaboradores a nivel mundial. Buena parte de su investigación fue financiada por National Science Foundation (NSF) de manera continua desde 1989. En 2012 se nombró a una lagartija patagónica en su honor, Phymaturus sinervoi.

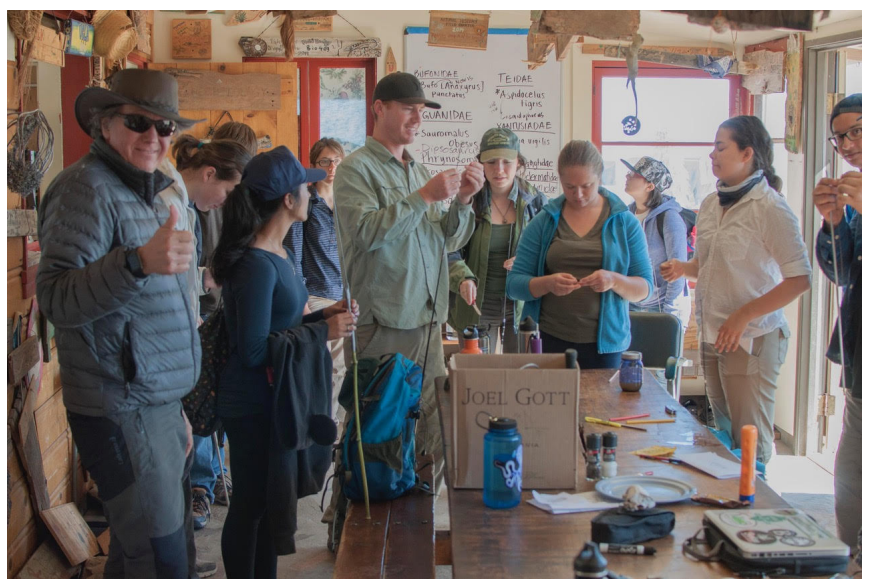

Figure 3. Barry Sinervo doing fieldwork and teaching herpetology in the Mojave Desert (April 2016). Photo: Rafael Lara.

Figura 3. Barry Sinervo haciendo trabajo de campo y enseñando herpetología en el desierto de Mojave (Abril 2016). Foto: Rafael Lara.

A pesar de sus continuos problemas de salud debidos al cáncer y a los tratamientos y cirugías nunca canceló su participación en eventos universitarios o científicos. Por ejemplo, en 2016 fue sobresaliente su participación en el congreso mundial de herpetología en China, pese a su voz y visión cedieron, impartió la conferencia magistral inaugural, pláticas regulares y un taller sobre ecofisiología y cambio climático. Posterior al congreso, realizó trabajo de campo, que culminó en la publicación de uno de sus artículos más recientes en Integrative Zoology: "Climate change, thermal niches, extinction risk and maternal-effect rescue of Toad-headed lizards, Phrynocephalus, in thermal extremes of the Arabian Peninsula to the Tibetan Plateau". A pesar de su diezmada salud, nunca perdió el sentido del humor y siempre sostuvo comunicación por correo electrónico con sus colegas y amigos hasta sus últimos días. Además, con entusiasmo siguió participando en sus clases de herpetología, mantuvo trabajo continuo con estudiantes y, por si fuera poco, escribió "Behavioral Genetics to Evolution" una obra gigantesca de 20 capítulos, 627 figuras y 465 videos narrados y explicados con animaciones; el cual sintetiza más de 30 años de investigación o como él lo mencionó en twitter: "... my epic journey through the field of Behavioral Ecology".

Barry Sinervo falleció el 15 de marzo de 2021 en California a la edad de 60 años después de una larga batalla contra el cáncer (carcinoma adenoide quístico). Le sobreviven su amorosa esposa Jeanie Vogelzang e hijo Ari Sinervo, quienes lo apoyaron y extrañan enormemente. Sin duda, la partida de Barry deja un gran vacío en la comunidad científica debido a sus inapreciables 
contribuciones académicas y trabajos pendientes, pero sobretodo deja un espacio imposible de llenar en quienes lo conocimos. Él dejó plantadas miles de semillas de amor a la naturaleza y al quehacer científico, y nos enseñó cómo la generosidad y el amor a lo que uno hace pueden ir de la mano de la rigurosidad y disciplina científica. Quienes tuvimos la suerte de trabajar con él, estamos agradecidos por sus enseñanzas y reconocemos sus valiosas contribuciones y estilo de vida inspiracional. Descansa en paz Dr. Lizardo.

\section{Publicaciones selectas}

Sinervo, B. 1990. The evolution of maternal investment in lizards: an experimental and comparative analysis of egg size and its effects on offspring performance. Evolution 44: 279-294.

Sinervo, B. \& S.C. Adolph. 1989. Thermal sensitivity of growthrate in hatchling Sceloporus lizards: environmental, behavioral and genetic aspects. Oecologia 78: 411-419.

Sinervo, B. \& D.F. DeNardo. 1996. Costs of reproduction in the wild: path analysis of natural selection and experimental tests of causation. Evolution 50:1299-1313.

Sinervo, B., P. Doughty, R.B. Huey \& K. Zamudio. 1992. Allometric engineering: a causal analysis of natural selection on offspring size. Science 258:1927-1930.
Sinervo, B. \& R.B. Huey. 1990. Allometric engineering - an experimental test of the causes of interpopulational differences in performance. Science 248:1106-1109.

Sinervo, B. \& P. Licht. 1991. Proximate constraints on the evolution of egg size, number, and total clutch mass in lizards. Science 252:1300-1302.

Sinervo, B. \& C.M. Lively. 1996. The rock-paper-scissors game and the evolution of alternative male strategies. Nature 380:240243.

Sinervo, B., F. Méndez-de la Cruz, D.B. Miles, B. Heulin, E. Bastiaans, M. Villagrán-Santa Cruz et al. 2010. Erosion of lizard diversity by climate change and altered thermal niches. Science 328:894-899.

Sinervo, B., D.B. Miles, W.A. Frankino, M. Klukowski \& D.F. DeNardo. 2000a. Testosterone, endurance, and darwinian fitness: Natural and sexual selection on the physiological bases of alternative male behaviors in side-blotched lizards. Hormones and Behavior 38:222-233.

Sinervo, B. \& E. Svensson. 2002. Correlational selection and the evolution of genomic architecture. Heredity, 89, 329-338.

Sinervo, B., E. Svensson \& T. Comendant. 200ob. Density cycles and an offspring quantity and quality game driven by natural selection. Nature 406:985-988.

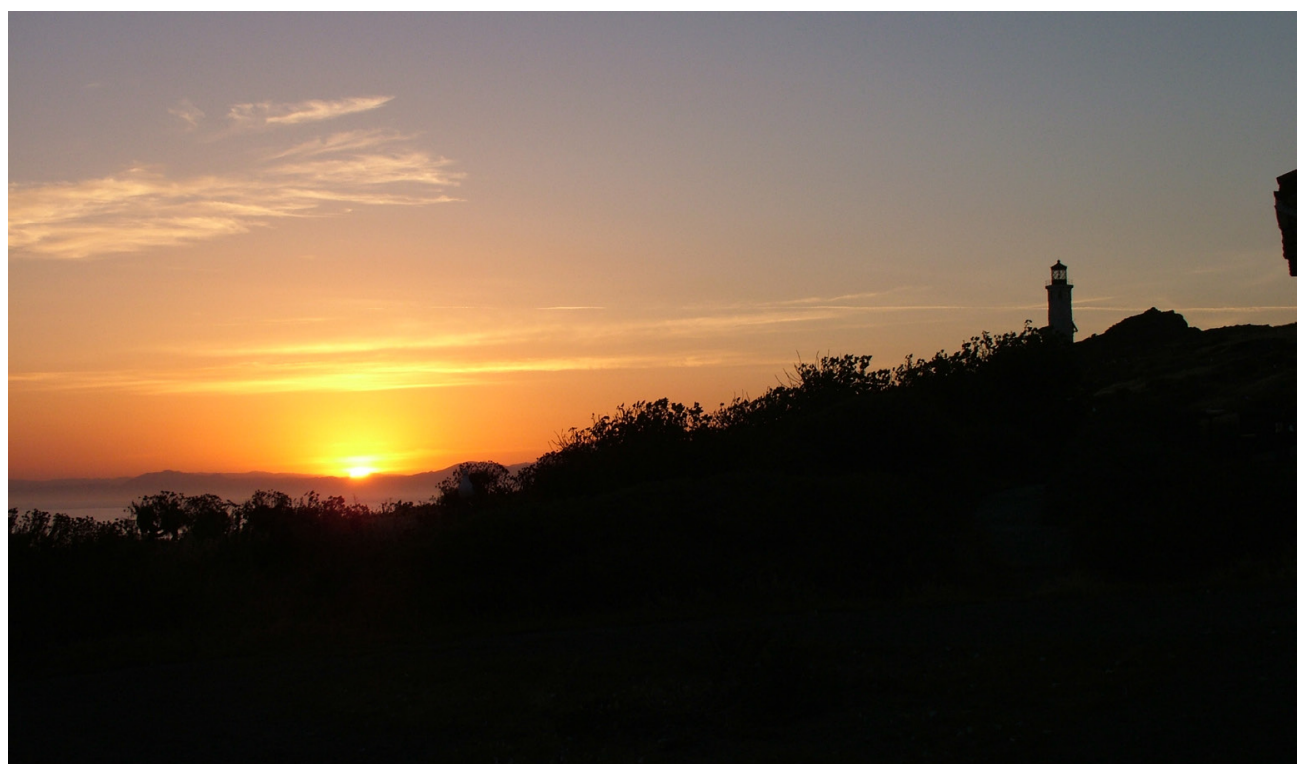

